\title{
Withdrawing neuroleptic treatment from people with dementia, does not result in a significant change to their behaviour, psychiatric symptoms, or quality of life
}

Ballard CG, Thomas A, Fossey J, et al. A 3-month, randomized, placebo-controlled, neuroleptic discontinuation study in 100 people with dementia: the neuropsychiatric inventory median cutoff is a predictor of clinical outcome. J Clin Psychiatry 2004;65:114-19.

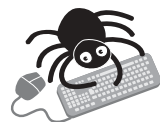

This article contains extra text on the EBMH website

\section{What are the effects of withdrawing neuroleptic treatment from people with dementia on behaviour, psychiatric} symptoms, and quality of life?

\section{METHODS}

L

Design: Randomised controlled trial.

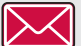

Allocation: Concealed.

Blinding: Double blinded.

Follow up period: Three months.

Setting: Residential care facilities in Newcastle and Oxford, UK

Patients: 100 people (76\% women) aged 65 years or older, living in a residential care facility with probable Alzheimer's

disease (National Institute of Neurological and Communicative diseases and Stroke/Alzheimer's Disease and Related Disorders Association criteria). Participants had a Clinical Dementia Rating scale (CDR) severity of stage 1 or greater, a Neuropsychiatric Inventory (NPI) score not greater than 7, and had taken neuroleptic drugs (thioridazine, chlorpromazine, haloperidol, trifluoperazine, or risperidone) for longer than three months (mean prescription time $>1$ year).

$\mathbf{R}$

Intervention: Neuroleptics (risperdone $0.5 \mathrm{mg}$, chlorpromazine $12.5 \mathrm{mg}$, thioridazine $12.5 \mathrm{mg}$, trifluoperazine $0.5 \mathrm{mg}$ or haloperidol $0.25 \mathrm{mg}$. Doses were kept as similar to original prescriptions as possible), or placebo for three months.

Find

Outcomes: Changes in behaviour and psychiatric symptoms (NPI); Quality of life (Dementia Care Mapping, (DCM)).

a Patient follow up: $82 \%$

\section{MAIN RESULTS}

There were no significant differences between treatment and placebo groups in NPI total score, psychiatric symptoms or quality of life (see http://www.ebmentalhealth.com/supplemental for table). Subgroup analysis of people with an NPI baseline score of above or below the median score of 14 found no significant differences in total NPI score, mood, psychosis, or quality of life. However, people with a baseline NPI score $\leqslant 14$ assigned to placebo experienced significantly less agitation compared with those receiving treatment $(p=0.018$, see notes).

For correspondence: Clive G Ballard, Wolfson Research Centre, Newcastle General Hospital, Westgate Road, Newcastle, UK; c.g.ballard@ncl.ac.uk Sources of funding: Research into Ageing, London, UK and Age Concern, London, UK.

\section{CONCLUSIONS}

Withdrawing neuroleptic treatment from people with dementia does not result in a significant change to their behaviour, psychiatric symptoms, or quality of life.

\section{NOTE}

The authors note that due to the sample size, the statistical power of this study is not great enough to detect significant differences in behavioural and psychiatric symptoms, so the results of the subgroup analyses should be treated with caution.

Commentary

D prem ementia with associated neuropsychiatric disturbance is widely prevalent in the nursing home population, and prescription of neuroleptics to these patients is in the order of $35 \%$. The evidence for their efficacy over placebo for the relief of agitation or periodic aggressive behaviour is limited. Evidence-based guidance on the merits of relatively short term usage has often been ignored. This clinical practice has resulted in the US in legislation to mandate review and discontinuation trial after a specified period. Ironically, there were few or no trial data available for neuroleptic discontinuation when the US OBRA 87 legislation was implemented in 1990.

One can speculate that neuroleptic prescribing behaviour is driven by understandable anxiety among both clinicians and carers about the risks of assault by, and also to, sufferers of dementia. However, concerns about side effects including parkinsonism and movement disorders, anticholinergic potential, increased fall risk, and worsening cognition cannot be ignored. These concerns have recently been sharpened by data suggesting a higher stroke risk in users of atypical drugs such as risperidone, with consequent Medicines Agency warnings about uncontrolled usage. This may have encouraged review of prescriptions, although discontinuation studies to date have been of limited value. Helpful publications differentiating risk among alternative antipsychotics are only now becoming available.

Discontinuation studies of neuroleptics in nursing home settings are particularly hard to do and have been methodologically limited by sample size, duration of withdrawal period, adequate blinding, and variability in outcome measures. This leaves clinicians with little usable data in a clinical situation, which Ballard and colleagues call "the almost impossible task of balancing the potential risk of adverse events with neuroleptic treatment against the potential exacerbation of behavioural symptoms if neuroleptics are discontinued". This familiar predicament is illuminated somewhat by findings of high study completion amongst those randomised to discontinuation, and by little evident worsening of behaviour, particularly among those with low levels of behavioural disturbance on the familiar, valid, and reliable Neuropsychiatric Inventory, prior to randomisation. With 100 patients randomised and a three month trial period completed, readers can see this as a useful study encouraging trials of neuroleptic withdrawal in patients with modest levels of behavioural disturbance.

Donald Mowat FRCP Edin, FRCGP, MRCPsych Consultant in Old Age Psychiatry, Royal Cornhill Hospital, Aberdeen, UK 